\title{
Paesaggi alla finestra: percezione delle trasformazioni e immaginazione delle alternative durante e dopo il lockdown
}

\author{
Gianluca Cepollaro \\ step-Scuola per il Governo del Territorio e del Paesaggio - Trentino School of Management. \\ gianluca.cepollaro@tsm.tn.it
}

Luca Mori

Dipartimento di Civiltà e Forme del Sapere, Post-Doc, Università di Pisa. moriluca@gmail.com

\begin{abstract}
La Convenzione europea del paesaggio (CEP), siglata a Firenze nel luglio 2000, promuove un'idea di paesaggio non come semplice sfondo delle attività umane, come un luogo di eccezionale bellezza da contemplare "dalla finestra", ma come "spazio di vita" delle popolazioni. Proprio nell'anno in cui si celebra il ventennale della sua ratifica, milioni di persone in tutto il mondo sono state costrette per diverse settimane, durante il periodo di lockdown dovuto all'emergenza pandemica, a non vivere i luoghi esterni alla propria abitazione e quindi, per cosi dire, a osservare i paesaggi ‘là fuori', a guardarli da 'finestre' reali o virtuali come gli schermi dei dispositivi digitali. In un progetto educativo svolto con i ragazzi delle scuole secondarie di secondo grado, il paesaggio diviene 'uno specchio di mondi possibili': esso non è solo l'occasione per rispecchiare lo stato d'animo che accompagna gli studenti durante l'isolamento, un oggetto 'fuori dalla finestra' sul quale si posa lo sguardo, ma è soprattutto occasione per riflettere sulla trasformazione delle percezioni e sull'immaginazione del futuro.
\end{abstract}

\section{Parole chiave}

Educazione al paesaggio, idea di paesaggio, percezione del paesaggio, Convenzione europea del paesaggio.

\begin{abstract}
The European Landscape Convention (ELC), also known as the Florence Convention, signed in 2000, promotes an idea of landscape as a "living space": landscape is not a background for human activities, is not a place of exceptional beauty to contemplate "from the window". In the 20th anniversary year of its ratification, millions of people around the world have lived through lockdowns for several weeks due to the coronavirus pandemic. Many have observed landscapes only 'out of there', looking at them from real or virtual 'windows'" (like the screens of digital devices). An educational project carried out with high school students during the last months, shows how landscape becomes 'a mirror of possible worlds'. It is not just an opportunity to reflect on 'the state of mind in isolation', it is not just an object 'outside the window', but an opportunity to reflect on the transformations of perceptions and imagination of the future.
\end{abstract}

\section{Parole chiave}

Landscape education, ideas of landscape, landscape perception, European Landscape Convention. 


\section{Introduzione}

Nello stesso anno in cui si celebrava il ventesimo anniversario della Convenzione europea del paesaggio (CEP), documento adottato a Strasburgo il 19 luglio 2000 che invita a non pensare il paesaggio come semplice sfondo delle attività umane, né come oggetto di mera visione e contemplazione distaccata, milioni di persone in tutto il mondo sono state costrette per diverse settimane a non attraversare i paesaggi esterni alla propria abitazione e quindi, per così dire, a osservarli 'dal di fuori', a guardarli perlopiù dalle finestre a loro disposizione, reali come quelle di casa, o virtuali come gli schermi di smartphone e computer.

La Convenzione ha giocato in questi anni un ruolo fondamentale nel promuovere la transizione del concetto di paesaggio da sfondo e decoro a 'spazio di vita', generando una sua estensione 'dall'essere visto all'essere vissuto' e connettendolo con l'idea di vivibilità. II paesaggio 'che siamo e viviamo' prende vita nell'incontro tra percezione, cognizione, memoria e risonanze emotive, dal quale scaturisce un'intonazione tra il visto, il pensato, il vissuto e l'immaginato (Tagliagambe, 2018, p. 15). II paesaggio, esito di un continuo e spontaneo processo di attribuzione di senso ai luoghi della vita, emerge nelle connessioni tra il mondo esterno ed il mondo interno (Morelli, 2011; Lingiardi, 2017) e si afferma metaforicamente come uno spazio relazionale, di prossimità, di coinvolgimento diretto, all'interno del quale le persone possono conversare, dialogare, negoziare, confliggere attorno ai tanti temi che da esso si dipanano. L'educazione al paesaggio, in particolare per le nuove generazioni, rappresenta un campo di straordinario interesse per affrontare temi estesi e controversi che hanno una forte connessione sulla qualità della vita attuale e futura.

\section{Emergenza sanitaria e crisi ecologica: una ricerca sull'educazione al paesaggio durante il lockdown}

Le misure di lockdown hanno determinato un repentino cambiamento delle abitudini a livello globale, con una conseguente riduzione degli spostamenti umani nello spazio e l'interruzione di moltissime attività impattanti sull'ambiente, che hanno provocato a loro volta trasformazioni rilevanti sia nei paesaggi, sia nei vissuti delle persone. Per la prima volta nella storia sono diventate osservabili, su scala planetaria, alcune trasformazioni legate non alla presenza di attività e al movimento degli esseri umani, ma alla loro assenza e all'interruzione degli spostamenti abituali.

L'emergenza causata dalla pandemia Covid-19 si è verificata, peraltro, in un'epoca di crescente consapevolezza e preoccupazione per un'altra emergenza, quella ecologica e climatica, legata all'insosteni- 
bilità dell'impatto delle attività umane sugli ecosistemi e, quindi, sulla buona vivibilità futura del pianeta per la nostra e per le altre specie.

Fin dagli anni Settanta, da quando si è iniziato espressamente a parlare di limiti dello sviluppo, un numero crescente di studi ha sottolineato l'esigenza di cambiare le nostre modalità di produzione e di consumo e, in generale, il nostro modo di stare al mondo e di sfruttare il pianeta, denunciando la cecità che ci porta a trattarlo come fonte inesauribile di risorse e come deposito interminabile di rifiuti (Meadows et al., 1972; Meadows et al., 2004). Le azioni intraprese fino ad oggi per affrontare la crisi ecologica e climatica sono state incerte e intempestive e non hanno portato a interruzioni delle abitudini e a cambiamenti dei comportamenti così bruschi e capillari come quelle determinate dalla pandemia Covid-19. Eppure gli effetti della crisi ecologica, che possiamo oggi interpretare metaforicamente come un'epidemia al rallentatore, sono altrettanto drammatici ed evidenti'. Senza alcun riferimento a relazioni certe e dirette tra crisi ecologica, riscaldamento climatico ed emergenza pandemica, preme sottolineare che già all'inizio degli anni Settanta, Gregory Bateson (1970) invocava una svolta epistemologica radicale a partire dal riconoscimento della connessione sistemica tra uomo e ambiente. Le immagini delle acque insolitamente trasparenti nei canali di Venezia, che hanno fatto il giro del mondo, documentano una delle tante conseguenze dell'interruzione delle abitudini umane a cui si è appena accennato; anche le acque del Po e di altri fiumi italiani sono diventate più limpide, destando l'attenzione dei principali quotidiani italiani; nelle grandi aree metropolitane e in alcune regioni, come la Lombardia, è stata registrata una significativa riduzione della concentrazione di sostanze inquinanti nell'aria, mentre altre immagini hanno documentato la riconquista di spazi da parte degli animali, come nel caso delle tartarughe che hanno ni- cervi e altri animali selvatici che vagavano tranquilli per le strade di paesi e città in cui prima non si erano mai spinti. Una delle testimonianze più significative - perché attesta la trasformazione di un paesaggio e, al tempo stesso, della percezione degli esseri umani che lo abitano - è arrivata dalla città indiana di Jalandhar, dove alcuni abitanti hanno condiviso via Twitter l'emozione di vedere per la prima volta dai tetti delle case, grazie al venir meno della cappa di smog sulla città, le vette del Dhauladar, a circa duecento chilometri di distanza: "Non ho mai visto scrive il 3 aprile l'ex giocatore indiano di cricket Harbhajan Singh - la vetta del Dhauladar dal tetto di casa mia a Jalandhar. Non avrei mai potuto immaginare che fosse possibile".

La documentazione di simili trasformazioni, con il sentimento di meraviglia che spesso le accompagna, ha stimolato una serie di confronti tra i paesaggi percepiti durante il lockdown e il loro stato precedente, ma ha anche alimentato riflessioni sul futuro dei paesaggi e sulle "lezioni" da trarre dalla pandemia, riflessioni che hanno trovato spazio in seminari, articoli, libri, appelli e lettere aperte ${ }^{2}$. Uno dei motivi ricorrenti degli interventi, esplicito o implicito, è in linea con quanto la Convenzione europea del paesaggio evidenzia già a partire dal "Preambolo". L'idea di paesaggio come bene comune (Settis, 2010; Parascandolo, Tanca, 2015; Gattullo, 2016), in particolare, trova fondamento nella dimensione dei processi e delle pratiche poste in atto dalle collettività per la sua salvaguardia, gestione e trasformazione, e pone importanti sfide alle politiche educative e formative necessarie per mettere le popolazioni nella condizione di partecipare attivamente alla costruzione e alla fruizione degli "spazi di vita” (Pedroli, Van Mansvelt, 2006; Castiglioni, 2010; Castiglioni, Cisani, 2020).

Nella lettera aperta di Roberto Danovaro e altri quattrocento scienziati, pubblicata dal quotidiano "Il Manifesto" il 20 maggio 2020, la prospettiva di lungo periodo viene declinata attraverso die- 
ci proposte per il 'dopo Covid-19', nelle quali - in modo diretto o indiretto - il paesaggio è sempre centrale. Più specificamente, diventa centrale l'invito a considerare in prospettiva sistemica il paesaggio e le scelte che lo riguardano, quasi a suggerire che la pandemia potrà avere "implicazioni sistemiche" sui nostri modelli di abitare ed attraversare i paesaggi non di per sé, ma soltanto se sapremo farla diventare un'occasione di apprendimento. Tra i punti in evidenza nel documento compaiono la prospettiva delle 'città verdi', la cessazione del consumo di suolo, la valorizzazione dei centri storici, la promozione dell'urban nature, gli investimenti sul turismo sostenibile, la lotta all'inquinamento, la cura della biodiversità e del "restauro ambientale", le politiche all'insegna del 'verde' e del 'blu'. A questi si accompagna la necessità di investire sulla ricerca e sulla formazione, insistendo sull'“insegnamento dei principi dell'ecologia e degli obiettivi di sviluppo sostenibile nella scuola dell'obbligo".

L'esperienza raccontata in queste pagine si inserisce nel filone di ricerca sull'educazione al paesaggio e si pone un triplice intento:

- valutare se la lettura del paesaggio può diventare una via d'accesso alla comprensione delle "implicazioni sistemiche della pandemia";

- rilevare se e come l'esperienza del lockdown ha inciso sulle percezioni, sulle rappresentazioni e sulle attribuzioni di valore concernenti il paesaggio in ragazze e ragazzi tra i 16 e i 19 anni;

- valutare se nuove intuizioni sul proprio legame con il paesaggio, suscitate dall'esperienza inedita del lockdown, hanno effetti sull'immaginazione degli studenti rivolta a definire i paesaggi futuri auspicabili per trarne alcune indicazioni per l'educazione al paesaggio.

\section{\#iorestoacasa. Il paesaggio, specchio di mondi possibili}

L'esperienza del lockdown ha offerto un'occasione unica per fare ricerca e formazione su alcune te-

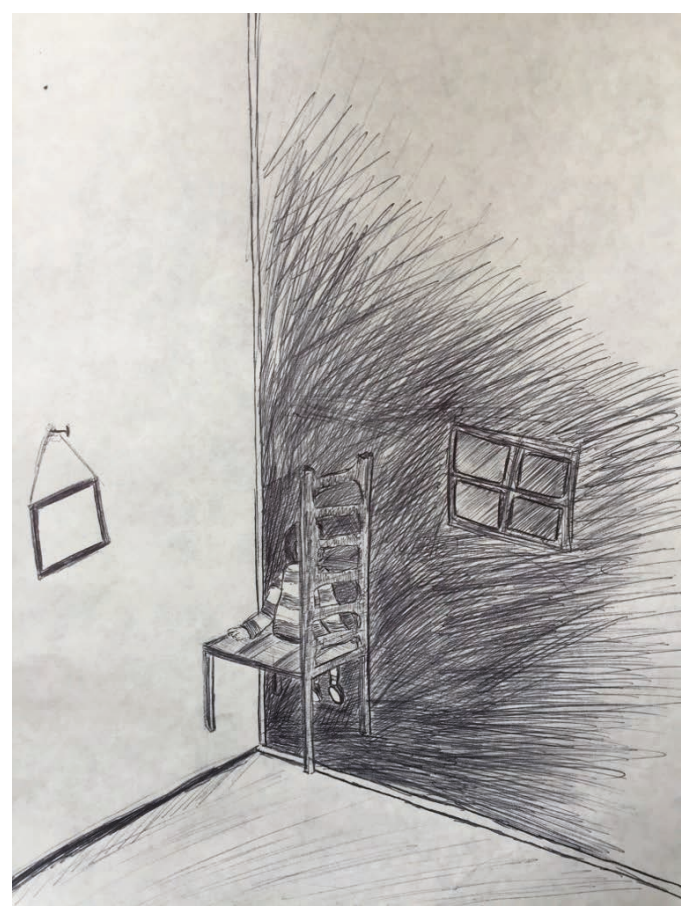

Fig. 1 - Francesco D'Angelo, Il vero nemico, penna su foglio A4, $21 \times 30 \mathrm{~cm}$.

matiche che emergono dalla nostra relazione con il paesaggio, proprio perché è questa relazione a subire un'improvvisa, inedita e drastica interruzione, almeno nei termini in cui abbiamo iniziato ad intendere il paesaggio come spazio di vita e non più come qualcosa da osservare a distanza, come un bel luogo da contemplare 'dalla finestra'.

Siamo in un momento in cui i giovani sono isolati e privati della partecipazione fisica alla comunità scolastica e al territorio, in una sospensione in cui il contatto con il mondo esterno può avvenire solo 'dalla finestra'. La didattica a distanza diviene l'unica modalità possibile di fare scuola, generando un profondo cambiamento dei luoghi dell'apprendimento, che si limitano sostanzialmente alla propria abitazione, mentre tutte le attività di comunicazione, condivisione, partecipazione avvengono esclusivamente tramite le tecnologie digitali. Gli schermi dei computer, dei telefonini, dei tablet e degli altri disposi- 

studenti, appartenenti ad undici classi di un Liceo delle Arti, che durante il periodo di lockdown hanno partecipato al progetto \#iorestoacasa. II paesaggio, specchio di mondi possibili.

Le attività, svolte secondo la modalità della didattica a distanza, si sono così articolate:

- conversazioni sul paesaggio con docenti ed esperti, di due ore per ciascuna classe nel corso delle quali alle studentesse e agli studenti, attraverso l'esperimento mentale dell'utopia, sono state poste alcune domande sulle trasformazioni percepite, probabili e auspicabili dei luoghi di vita durante e dopo l'emergenza pandemica (Mori, 2020);

- conferenze online di esperti per la presentazione del progetto finalizzato all'elaborazione dell'idea di paesaggio nel periodo del lockdown;

- attività con le classi e con i docenti per individuare alcune forme di rappresentazione (racconti, disegni, fotografie, video, performance) del rapporto tra i ragazzi e i loro "spazi di vita" durante il lockdown. Per la realizzazione dei prodotti, le classi sono state affiancate da alcuni esperti al fine di valorizzare le connessioni con i linguaggi propri delle diverse materie caratterizzanti il curriculum petenze di espressione e rappresentazione degli studenti;

- presentazione pubblica dei lavori svolti con il coinvolgimento delle undici classi, del corpo docente e di alcuni rappresentanti delle istituzioni culturali del territorio (Comune di Trento, tsm-Trentino School of Management, Fondazione Museo storico del Trentino, MUSE-Museo delle Scienze di Trento).

Le domande poste durante le conversazioni, volte ad indagare le trasformazioni percepite, gli scenari probabili e quelli auspicabili, erano le seguenti:

- notate dei cambiamenti significativi nel paesaggio 'esterno' che osservate dalla finestra o che attraversate durante le brevi e limitate uscite concesse in questi giorni?

- Quali cambiamenti nel vostro mondo "interno" sentite più rilevanti durante questi giorni di lockdown?

- Pensando 'al prima' e 'all'adesso", come vorreste che fosse 'il dopo'?

- Quali cambiamenti in meglio auspicate, tenendo conto di quanto avete pensato e vissuto durante il lockdown?

L'analisi delle conversazioni e delle rappresentazioni raccolte è avvenuta in riferimento sia al loro valore di testimoniare un presente inaspettato quanto drammatico, sia al loro valore proiettivo di indicare trasformazioni attese e auspicabili per 'il dopo' pandemia ${ }^{3}$.

\section{Paesaggi alla finestra: trasformazioni percepite}

Uno sguardo d'insieme alle percezioni del paesaggio del lockdown, emerse nelle conversazioni, permette di individuare i seguenti temi ricorrenti:

- le trasformazioni della dimensione sonora e cromatica dell'esperienza del paesaggio, nonché la presenza e il movimento degli animali;

- il rallentamento dei ritmi e l'interruzione dei movimenti frenetici che permettono di rivalutare alcuni spazi domestici e in prossimità dell'abitazio- 

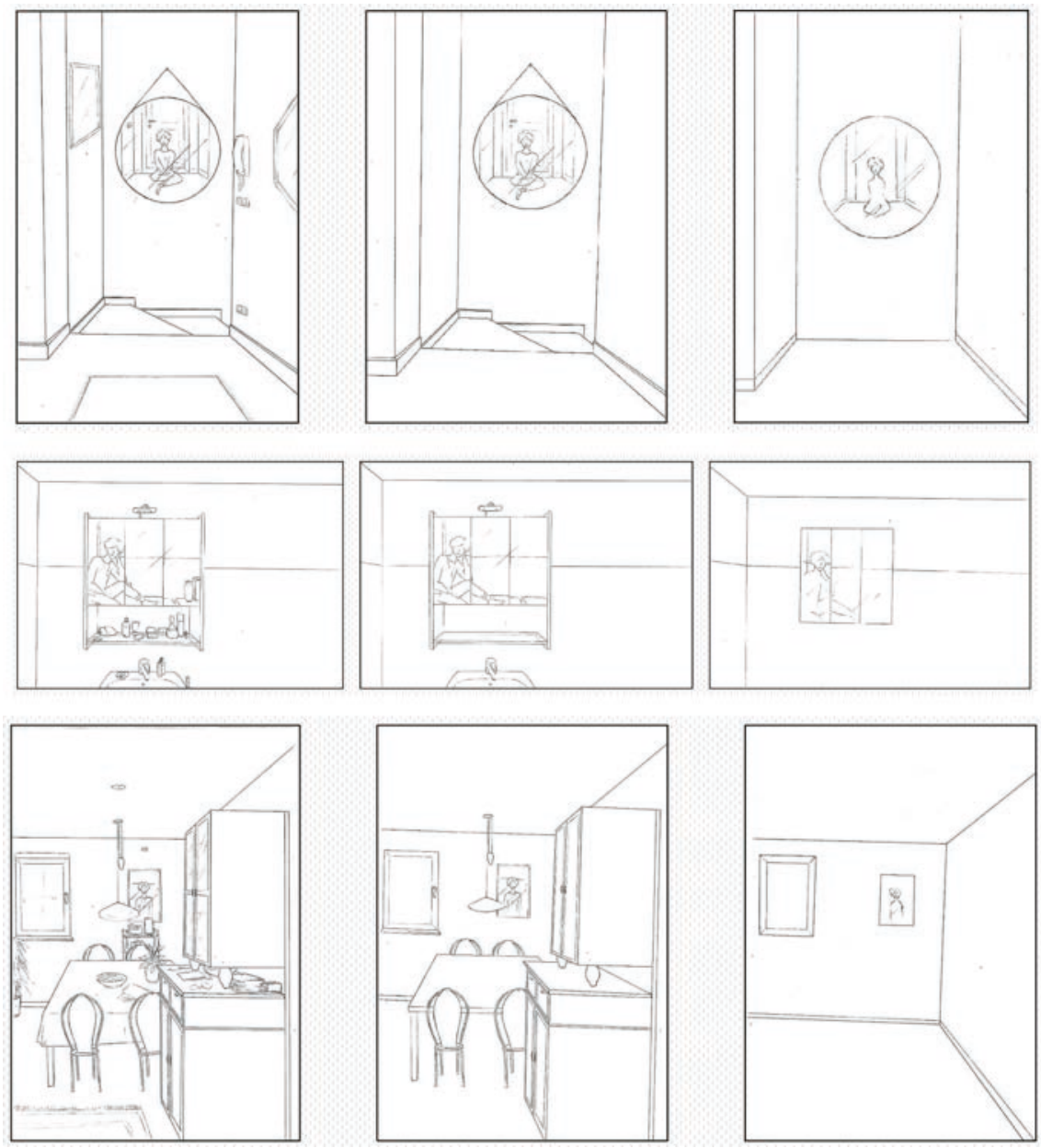

Fig. 2 - Leonardo Giuliani, Senza titolo, penna su carta. 
ne e, al tempo stesso, di sperimentare nuove attività;

- la sensazione di avere 'aperto gli occhi' su aspetti del paesaggio e dell'esistenza a cui prima non si prestava attenzione, come se l'immersione precedente nel paesaggio fosse in parte "anestetizzata"4

Parliamo di paesaggi alla finestra in riferimento al fatto che il lockdown ha comportato un'interruzione dell'esperienza consueta di attraversamento ed immersione del paesaggio, costringendo a guardarlo per molto tempo a distanza, dalle finestre reali della propria casa o da quelle virtuali dei dispositivi tecnologici. Cosa si può dire e conoscere in questa posizione?

Per Leon Battista Alberti la finestra è l'emblema di una visione frontale che permette di conoscere il mondo mettendolo in prospettiva, riproducendo le tre dimensioni su un piano bidimensionale, puntando al risultato ottimale della riproduzione altamente definita. C'è però anche la finestra di Magritte, che riprende la pretesa della finestra albertiana di rappresentare fedelmente il reale segnalandone al tempo stesso il limite, dando sottile evidenza alla sua cornice e quindi alla persistente, insopprimibile differenza tra ogni rappresentazione frontale e il suo referente: il che, in termini di paesaggio, significa che nessun paesaggio coincide con la sua rappresentazione frontale, anche se siamo esposti alla confusione tra i due piani. II nostro lavoro sui paesaggi alle finestre, siano essi reali o virtuali, ha tenuto presente l'esigenza fondamentale di dare risalto alle cornici attraverso le quali consideriamo i paesaggi, connettendo il piano delle percezioni a quello dei significati (lacono, 2010).

Prima di riportare alcune considerazioni esemplari sui punti sopra elencati, è bene precisare che non in tutti i luoghi sono stati rilevati gli stessi cambiamenti o la stessa intensità di cambiamento: insomma, c'è chi ha notato grandi differenze nel proprio ha viste poche o nessuna. In genere, ne ha viste meno chi abita in posti isolati in montagna o chi abita in luoghi in cui determinate attività lavorative non sono state interrotte, come Celeste in Val di Non, che osserva: "sento [comunque] molto la presenza dei trattori, che vanno a buttare il veleno: questa cosa non è cambiata, perché i contadini girano lo stesso". Iniziando dalla dimensione sonora dell'esperienza del paesaggio, in generale è la riduzione del rumore del traffico veicolare a colpire maggiormente. Nicolò, ad esempio, che vive vicino all'autostrada, ha fatto esperienza di un 'silenzio mai sentito" prima. Maddalena, che non abita in prossimità della città, ma vede comunque in lontananza e dall'alto l'autostrada, rileva la riduzione delle luci delle auto e soprattutto "meno rumori", perché il traffico dell'A22 abitualmente "rimbomba tutto nella valle". Lo conferma Ottavio, che vive a Folgaria: "Un giorno, mi sembra la settimana scorsa, ho deciso di fare una passeggiata dove prediligo, sopra Castel Beseno (Calliano). Mi ricordo che c'era sempre un rumore di sottofondo, che era l'autostrada, e mi sono seduto in una panchina e la strada era deserta: il sole, il profumo, il bosco, era come se tutto fosse nuovo". Adriana abita invece vicino a un lago e la sua testimonianza è la seguente: "tra casa mia e il lago c'è la statale e ci sono stati dei giorni in cui c'era talmente silenzio che si sentivano le anatre a distanza. La statale era sempre piena di macchine e ora è come il deserto, ed è bellissimo".

Notevole anche il caso segnalato da una ragazza che vive a Pinzolo, che si è accorta di poter sentire meglio lo scorrere del fiume Sarca "in fondo al paese": anche nel suo caso si può dire che "non l'aveva mai sentito così". Un ragazzo che vive in un piccolo paese di circa duemila abitanti in Val di Fiemme racconta di avere vissuto l'esperienza insolita di sentire, durante il tragitto a piedi verso un negozio, il rumore dei propri passi e del proprio respiro e di avere sentito in modo diverso anche i propri pensieri, in un paesaggio che sembrava quasi post-apocalittico. 
Dal momento che "il livello sonoro medio si è abbassato", come osserva Claudio, diventa possibile accorgersi di suoni e rumori che prima non si udivano e che, in generale, sono riconducibili al movimento della natura (fiumi, uccelli, vento, ecc.). Nel riscoprire il paesaggio sonoro viene attribuito un valore anche al silenzio non inteso come assenza 'di', ma come occasione 'per' apprezzare nuovi suoni e rumori. In questa riscoperta vi è la possibilità di svincolare l'idea di paesaggio come esito di un "sistema di percezione dominante" centrato sulla vista e caratterizzato dalla frontalità e dalla distanza (Vitale, 2015, pp. 8-12).

Anche sul piano cromatico l'esperienza del paesaggio appare trasformata. Raffaele, ad esempio, oltre alla riduzione dell'inquinamento acustico, ha notato un'aria quasi più fresca, colori più vividi e giardini pubblici più rigogliosi. Un'osservazione di Marta solleva un dubbio al riguardo: potrebbe darsi che il paesaggio appaia più vivo e colorato perché si fa più attenzione a quel che abbiamo attorno. L'allentamento delle routine, del 'troppo', del saturo e del caotico, sembra risvegliare ed alimentare nei ragazzi una rinnovata attenzione, una capacità espansa di tendere verso qualcosa di nuovo, prima non considerato o non accessibile, che permette di avere una diversa consapevolezza ed un diverso uso dei luoghi. II legame tra l'atteggiamento osservativo e la trasformazione dell'oggetto osservato appare chiaro nella dichiarazione di Lia: "Da me in questo periodo si vedono molto di più le stelle, ho avuto anche il tempo di dedicarmi ad osservarle". Non mancano tuttavia le considerazioni che danno una ragione oggettiva delle trasformazioni rilevate: Filippo, ad esempio, ha notato che il prato vicino a casa sua è più colorato perché è pieno di fiori, cosa che prima non accadeva perché erano molte le persone che lo attraversavano e quindi lo calpestavano o andavano lì per fare picnic, schiacciando erba e fiori sotto le tovaglie; Silvana, invece, abitando in una posizione da cui è possibile vedere Rovereto dall'alto, ha notato che la città di mattina non appare sovrastata e in

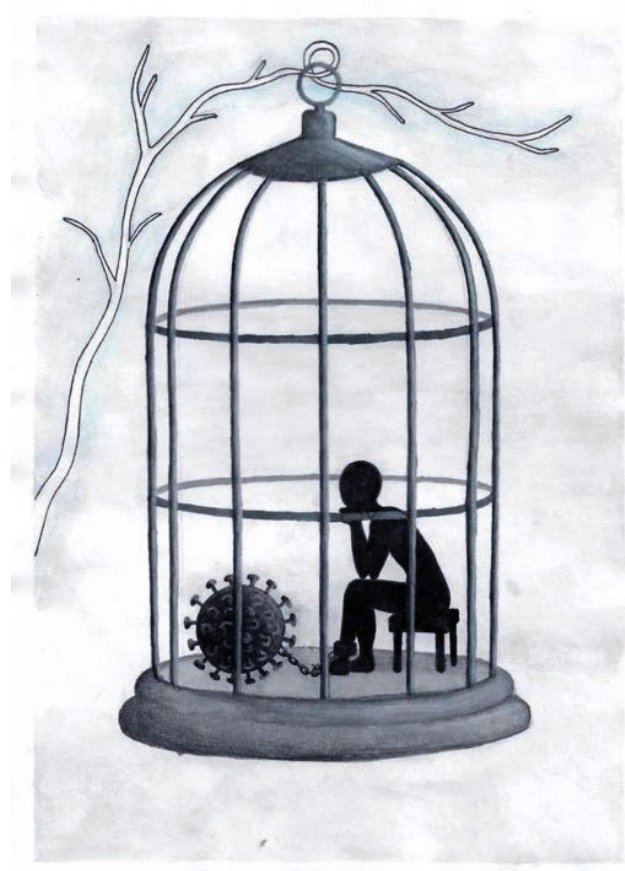

Fig. 3 - Sasha Gilodi, Senza titolo, penna su carta, A4, $21 \times 30 \mathrm{~cm}$.

parte nascosta "da una specie di foschia", come accadeva precedentemente.

II ritrarsi della presenza e dei movimenti umani lascia spazio a quelli degli animali: c'è chi ha visto più insetti in circolazione (addirittura più sciamature), chi ha osservato e sentito gli uccelli e il loro canto, e chi ha scorto animali selvatici in circolazione. Francesca, ad esempio, racconta di avere visto giocare per la prima volta un capriolo: "non avevo mai visto qualcosa di questo tipo e proprio ora che sono ricominciati i lavori agricoli è scomparso". In questi casi è chiaro che ciò che si osserva attorno a sé dipende dalla posizione e dal moto dell'osservatore.

L'interruzione forzata delle occupazioni precedenti consente di scoprirne di nuove: c'è chi ha fatto "ritorno alla manualità" coltivando un orto in giardino; c'è chi ha iniziato a leggere libri diversi da quelli scolastici e chi addirittura si è meravigliata di se stessa per avere provato il piacere di leggere, trovando 

tare e attraversare i paesaggi e il sentimento di sé. legato al sentimento del tempo e dello spazio; in altri termini la scoperta riguarda il fatto che il modo in cui "mettiamo al mondo il paesaggio" (secondo determinati modelli di abitarlo e attraversarlo, ad esempio) incide profondamente sul modo in cui il paesaggio "ci mette al mondo", secondo un principio di circolarità in cui sono in gioco, assieme a noi umani, le altre specie e gli stati dinamici degli ecosistemi in cui viviamo (Cepollaro, Mori, 2018).

Tale sensazione di "riscoperta" si dipana dal macro al micro, spaziando dalla dimensione della valle dell'Adige percepita in modo inedito agli spazi domestici: ad esempio, c'è chi ha iniziato ad utilizzare un poggiolo che precedentemente ignorava, riscoprendone con sorpresa la funzione dimenticata; c'è chi sente la mancanza di un terrazzo e di un giardino e chi invece, avendo l'uno o l'altro, ammette di averli fortemente rivalutati durante il lockdown. In generale si sente l'esigenza e si riconosce il valore della polifunzionalità degli spazi abitativi e della loro apertura all'ambiente esterno.

Le scoperte percettive e cognitive sembrano convergere ed essere riassumibili nella metafora dell"'aprire gli occhi': si ha la sensazione di vedere in faccia quel che accade, quel che è, come se esso rienza limite e traumatica come quella del lockdown $n^{5}$. Adriano, ad esempio, esprime questa sensazione, facendo riferimento alla propria esperienza, ma anche a quella degli abitanti del suo paese subito dopo il lockdown, che prima passeggiavano raramente in certe zone attorno al paese: "Ė una scoperta della natura da parte di chi prima ha avuto un contatto superficiale, da parte di persone che non avevano l'esigenza di stringere un rapporto con il paesaggio, con l'ambiente; forse in questa quarantena gli si sono aperti un po' gli occhi verso il mondo". Lo conferma Viola: "lo invece potrei dire che sul fatto di vedere la natura qualcosa è cambiato. Anche solo vedere le montagne vicino a casa mia: non è una cosa nuova, ma mi sono accorta che le vedevo in modo diverso, prima. [E anche gli altri abitanti del paese] almeno si sono resi conto [di quello che abbiamo]; nel mio paese veramente nessuno andava in giro per strada e adesso, forse, si sono resi conto in questa situazione [di come è importante] uscire. Forse è un'occasione di guardare in un modo diverso il nostro paese, la natura e tutto".

L'esperienza del lockdown, ancora una volta, porta a scoprire o a riscoprire il valore dei paesaggi abituali e alimenta una nuova attenzione ai paesaggi di prossimità. I paesaggi "perduti”, causa le restrizioni legate al movimento delle persone, sono sostituiti dai paesaggi di prossimità spesso poco considerati proprio dai giovani perché ritenuti "ovvi" e a portata di mano. Raffaele commenta in chat: "Ci stiamo comportando come se avessimo appena scoperto l'acqua calda: tutte queste cose di cui parliamo sono sempre esistite, molto prima della quarantena, e prima dell'umanità. Eravamo ciechi, e ora tutto questo ci sta facendo aprire gli occhi. Forse sarebbe il caso di non dimenticare, o rischiamo di non riuscire più a vederle”. Ariel si dice d'accordo con chi parla di "apertura degli occhi": "questo periodo mi ha aperto un po' gli occhi per quello che sta succedendo, per quello che stiamo vivendo io e la mia famiglia, per le abitudini che avevo prima e non ho più. [...] Di sicuro 
non correrò come prima, per gli appuntamenti e per le cose che dovremo fare". Arianna però al riguardo ha dei dubbi, perché a suo avviso "di solito si tende a dimenticare quello che si impara, dopo avere superato un ostacolo".

\section{I paesaggi dopo l'emergenza: scenari probabili}

La considerazione di Arianna ci porta all'analisi degli scenari futuri ritenuti probabili. Benché la condizione di relativa stasi abbia "aperto gli occhi" a tanti, in molti dichiarano l'esigenza di attraversare il paesaggio e di sentire quel "ricambio di energia" che si prova incontrando persone diverse e andando in luoghi diversi. Se qualcuno ha sottolineato che la pausa dalla frenesia consueta può stimolare la creatività, qualcun altro ha notato che la staticità alimenta anche la pigrizia e riduce la creatività. Si sente l'esigenza, pertanto, di tornare a muoversi; ma come ci muoveremo? Terremo conto, nel riprendere i nostri movimenti, delle scoperte fatte durante il lockdown?

Riformuliamo la domanda mettendola a distanza dai vissuti personali e considerando il caso esemplare del cittadino di Jalandhar che ha scoperto, con grande meraviglia, di poter vedere dal tetto di casa le vette del Dhauladar. Quando il lockdown finirà e la città riprenderà i ritmi consueti, rialzando la cortina di smog tra se stessa e l'orizzonte e il colore del cielo, quell'abitante si rassegnerà facilmente a rinunciare alla bellezza possibile che pure ha intravisto inaspettatamente?

Generalizzando e ampliando la domanda ci si potrebbe chiedere se saremo in grado di tornare a muoverci in modo da non perdere la bellezza che sarebbe, nonostante tutto, a nostra portata di mano, e se sapremo cambiare i nostri modi di abitare, costruire e attraversare i paesaggi, in modo che la nostra presenza e il nostro passaggio non ne compromettano la vivibilità e la bellezza, qualità che rendono al tempo stesso possibile e più godibile la vita. Se non ne fossimo capaci, ci ritroveremmo in una condizione analoga a quella del mitico Tantalo, il cui supplizio con-

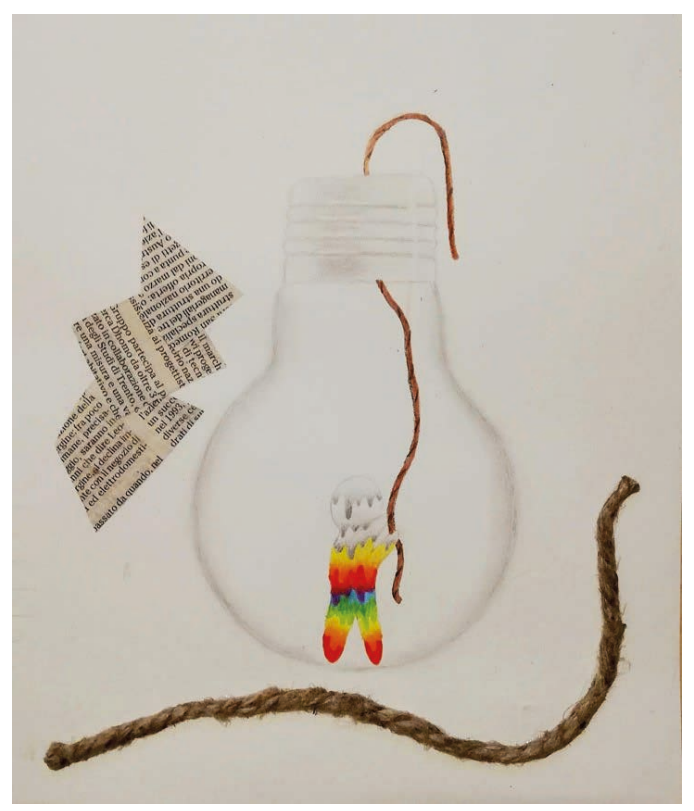

Fig. 4 - Denise Marinelli, La Speranza, carta di giornale e acrilici, $24 \times 20 \mathrm{~cm}$.

sisteva nel trovarsi immerso nell'acqua, in prossimità di un albero ricco di frutti; essendo affamato e assetato, non appena si muoveva per bere e per raccogliere i frutti che erano a sua portata di mano, l'acqua e i frutti si ritraevano diventando irraggiungibili, proprio in ragione del suo movimento. A proposito del cittadino di Jalandhar, dunque, c'è chi dice che proverà rabbia e dispiacere, ma che si dovrà rassegnare: "probabilmente, una volta tornati alla normalità, nessuno penserà più al paesaggio e al resto"; "torneremo a pensare ciò che pensavamo prima"; "non ci farà neanche più caso"; "mi piacerebbe che le persone imparassero qualcosa da tutto questo, ma non credo che succederà, perché tendiamo ad ignorare i nostri errori"; "non mi aspetto grandi cambiamenti positivi; anzi, la frenesia sarà triplicata per recuperare il tempo in cui siamo stati bloccati". Adelaide ritiene che senza un "grande disastro del genere, nessuno si sarebbe dato una svegliata", ma teme che "le persone torneranno ad adagiarsi nella loro quotidianità di prima". E porta una prova: "è vero che in questi mesi abbiamo vi- 
struzione di quel che ha per riuscire a darsi dei limiti (Georgescu-Roegen, 1982, p. 75) ${ }^{6}$.

Concludiamo la rassegna delle posizioni con alcune voci che ritengono invece probabile un cambiamento positivo: secondo Celeste "non sarà più come prima, perché questa quarantena è stata utile per pensare" e, in primis, si darà "più importanza agli affetti e alle relazioni umane"; anche secondo Ilaria difficilmente le cose torneranno come prima, soprattutto perché l'esperienza del lockdown ha dato prova della "forza dell'azione collettiva", mostrando che impegnarsi tutti insieme per un cambiamento "può fare veramente la differenza"; secondo Maddalena, ci sono molte persone che hanno apprezzato l'uscita dalla frenesia e dai ritmi precedenti e che forse continueranno a desiderare di vivere "senza tutte quelle scadenze e quegli impegni, [con la sensazione che] se restiamo fermi un paio d'ore tutti gli altri vanno avanti e noi rimaniamo indietro".

Daniela riporta, per concludere, anche un caso di piccolo cambiamento innescato durante il lockdown a Mattarello, "in un quartiere abbastanza chiuso di stradine": "mi sono accorta in questo periodo che passano meno macchine e i bambini si sono riappropriati di queste stradine. Hanno riscoperto vecchi giochi di strada. C'è un piccolo fazzoletto di terra abbandonata: è venuta ai bambini l'idea di dire ai genitori di comprare quel pezzo di terra abbandonata per fare un piccolo parco nel quartiere".

Dalle aspettative degli studenti emergono spesso un bisogno e un desiderio di comunità e di socialità: la maggiore consapevolezza della frammentazione delle relazioni e dell'indebolimento del legame sociale, esistenti già da prima del distanziamento fisico imposto dal lockdown, fa sperare nella riconquista di spazi di aggregazione e nella creazione di luoghi di dialogo e scambio.

\section{I paesaggi dopo l'emergenza: scenari auspicabili}

Per riassumere il paradossale scarto tra i paesaggi percepiti e desiderati durante il lockdown e quel-

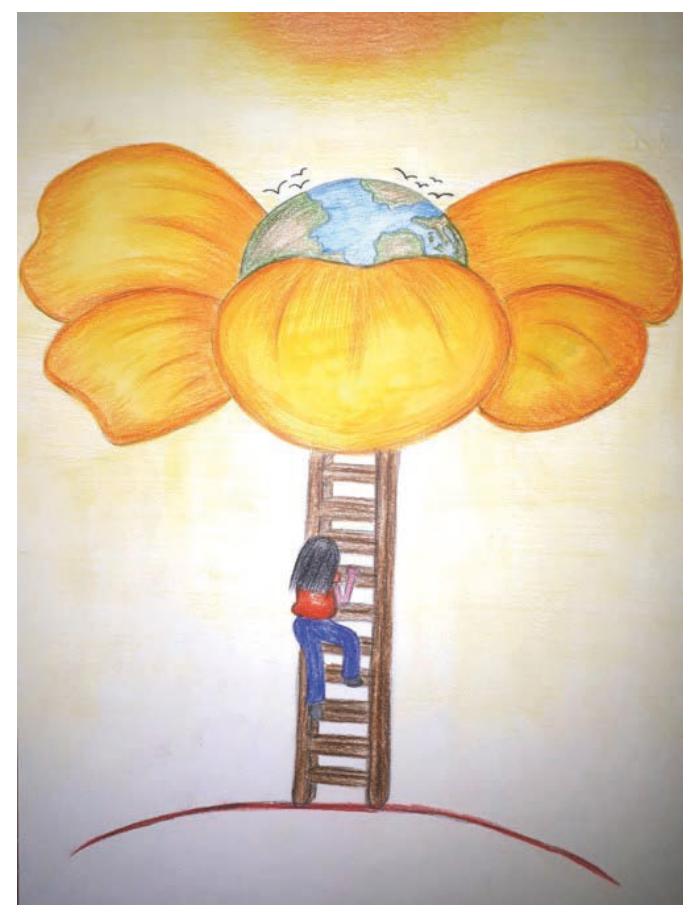

Fig. 6 - Serena Graziadei, Senza titolo, matite colorate.

li ritenuti probabili in seguito abbiamo fatto riferimento al mito di Tantalo, che non riesce a muoversi senza allontanare da sé i "frutti del paesaggio" a cui aspira. La nostra condizione non è alla lettera quella di Tantalo, ma sul piano metaforico la sua condizione assomiglia alla nostra: I'auspicio ricavabile dalla ricerca presentata in queste pagine è che lo shock provocato dall'emergenza in corso carichi di tensione utopica l'immaginazione educativa, architettonico-urbanistica e politica di tanti, o almeno di una significativa "minoranza attiva" capace di ispirare cambiamenti che permettano alla nostra specie di esplorare i propri margini di miglioramento nel rapporto con i propri "spazi di vita".

Molte ragazze e molti ragazzi sono d'accordo con una dichiarazione di Stefano Boeri, secondo la quale il ritorno alla normalità, dopo l'esperienza fatta, sarebbe "la cosa più preoccupante": "Qui si tratta davvero di cambiare il modo di vivere delle città"7. 
di "visualizzare" al meglio ciò che si desidera. C'è chi osserva, a questo punto, che se la volontà di cambiamento resta inespressa, oppure confusa e vaga, è molto facile ricadere nei modelli e nei paradigmi già esistenti; se invece la volontà di cambiamento dei giovani trova espressione in immagini e modelli condivisibili e accattivanti, allora queste immagini e questi modelli potrebbero diventare dei punti di riferimento e delle fonti di ispirazione per dei cambiamenti possibili.

Occorre dare forma al desiderio e all'immaginazione, affinché qualcosa che ancora non esiste possa iniziare a esistere, ed è qui che affiora con forza un'altra possibile funzione - e responsabilità - dell'educazione al paesaggio: mostrare come dare forma ai pensieri e alimentare l'immaginazione attraverso la conversazione e come dare forma all'immaginazione mediante il sapere tecnico.

Dalle idee circa l'inesistente immaginato e desiderato (e da quel che si cerca al riguardo: sostenibilità, crescita, buona vivibilità o una loro combinazione) dipende la possibilità di intravedere nuovi mondi. Per le giovani generazioni non mancano le ragioni di preoccupazione, ansia e paura per l'esistente, ma nello stesso tempo appaiono evidenti anche una tensione e una disponibilità inedita a pensare un futuro diverso.

\section{Conclusioni: alcuni spunti dall'esperienza di lock- down per l'educazione dal paesaggio}

La ricerca ha permesso di rilevare il forte impatto emotivo e cognitivo che l'esperienza del lockdown ha avuto per i giovani studenti coinvolti. L'esperienza estrema, mentre ha inciso chiaramente in modo molto forte sulle loro percezioni, rappresentazioni e attribuzioni di valore concernenti il paesaggio, ha permesso ai ragazzi di accedere ad una più profonda conoscenza della connessione e della ricorsività tra "mondo interno e mondo esterno", tra mindscape e landscape, nella costruzione dei paesaggi della propria vita.

Il carattere di un'esperienza limite e l'elaborazione avvenuta in termini educativi hanno aiutato ad 'aprire gli occhi' sui nessi sistemici tra la configurazione dei paesaggi di prossimità, i modi e ritmi dell'abitarli e dell'attraversarli, il senso del tempo e dello spazio, il sentimento di sé e le possibilità di movimento per gli esseri umani e le altre specie. II poter cogliere tali nessi con insolita evidenza costituisce per chi si occupa di educazione al paesaggio un'occasione per tenerli presenti anche in futuro e per scoprirne altri. Ciò non significa però, dal punto di vista degli studenti, che la pandemia possa avere di per sé effetti duraturi sul paesaggio, perché molti ragazzi temono che a un certo punto, superata l'emergenza, 'tutto tornerà come prima', sotto la spinta inerziale delle condotte di vita precedenti. Ciò che può fare la differenza è l'educazione dello sguardo sulle trasformazioni dei paesaggi e sulla loro complessità e, in questa prospettiva, ciò che è accaduto durante la pandemia potrebbe essere ripreso e analizzato anche quando l'emergenza sarà superata. L'elaborazione dell'esperienza del lockdown ha permesso agli studenti di individuare alcune trasformazioni legate alla pandemia, partendo dalla riflessione sugli spazi di prossimità per arrivare ad una nuova consapevolezza del proprio legame con il paesaggio. Affinché tali effetti possano durare nel tempo, tuttavia, si ritiene necessario mantenere viva ta- 
le consapevolezza, cosa che potrà essere fatta anche considerando la lezione della pandemia negli approcci e nei programmi educativi. Le testimonianze dei giovani, in particolare conversazioni e riflessioni sull'esperienza della primavera del 2020, non più in senso stretto riproducibile, rappresentano quindi materiali unici, che potrebbero essere utilizzati in un futuro programma di educazione alla lettura sistemica dei paesaggi e delle loro trasformazioni.

Le nuove intuizioni sul legame con il proprio spazio di vita sollecitate dall'esperienza di lockdown mostrano come l'educazione al paesaggio possa svolgere il fondamentale ruolo di allenare a pensare nuovi mondi possibili, qualora riesca a coniugare lo studio del passato e l'analisi del presente con l'immaginazione di quel che ancora non esiste, nella declinazione del futuro auspicabile. La sfida diventa pertanto quella di tenere traccia della lezione della pandemia (con i suoi effetti più e meno duraturi) sia nei programmi di educazione al paesaggio, sia nella progettazione futura dei paesaggi: come contributo al compito questo articolo ha cercato di documentare intuizioni e propositi maturati in settimane che non avevano precedenti nella storia mondiale.

\section{Note}

${ }^{1}$ Cfr. L'emergenza climatica è una pandemia al rallentatore (Testa, 11 maggio 2020).

2 Tra le recenti pubblicazioni sull'idea che la città sia da ripensare come bene comune, cfr. La città per l'uomo ai tempi del Covid-19 (Cannata, 2020); Fabbricare fiducia al tempo del Covid19 e oltre (Sciascia, 2020).

${ }^{3}$ Quando, citando le dichiarazioni dei ragazzi, mettiamo delle frasi tra virgolette, riportiamo fedelmente la trascrizione del parlato, utile talvolta a cogliere le sfumature e il coinvolgimento emotivo, che non sarebbero riproducibili nel discorso indiretto. I disegni, le fotografie e le immagini presentate in queste pagine sono frutto di una selezione finalizzata ad accompagnare in termini evocativi le riflessioni e le conversazioni contenute nel presente contributo. ${ }^{4}$ II riferimento implicito ad una percezione abituale anestetizzata è in linea con le considerazioni di Andy Hargreaves riportate da Zygmunt Bauman in una sua intervista sull'identità, a proposito del fatto che "negli aeroporti e in altri spazi pubblici gli individui col telefono cellulare e l'auricolare camminano qua e là, parlando ad alta voce da soli, come schizofrenici paranoici, incuranti di ciò che sta loro intorno". Cfr. (Hargreaves, 2003, p. 25; Bauman, 2009, p. 26)

${ }^{5}$ Richiamiamo qui l'idea di "situazione limite" proposta da Karl Jaspers, cioè di una situazione che pone i soggetti di fronte alla propria finitezza e all'esigenza di cercare un "punto d'appoggio" (la morte è una di queste situazioni): le situazioni limite hanno in comune il fatto che in esse diventa evidente che "tutto scorre, è preso nel moto irrequieto dell'essere-posto-in-forse, tutto è relativo, finito, scisso in contrari, non è mai il tutto, l'assoluto, l'essenziale" (Jaspers, 1950, p. 267). Pur essendo in senso stretto insopportabili per chi le vive, Jaspers aggiunge che in pratica "abbiamo quasi sempre un punto d'appoggio di fronte alla situazione limite", e il punto d'appoggio trovato mostra quali forze agiscono all'interno di un essere umano. 
01

2021
${ }^{6}$ L'espressione "comodità esosomatiche" fa riferimento alle comodità determinate da tutto ciò che costruiamo, "esterno" al nostro corpo, per ridurre il tempo o la fatica che impiegheremmo facendo da soli, con le forze del nostro corpo: esemplare in questo senso è la macchinina sui campi da golf, che rende "più comodo" un tragitto che potrebbe essere fatto tranquillamente a piedi; a costituire un problema, dal suo punto di vista, è l'eccesso di comodità superflue che costruiamo e alle quali ci abituiamo. ${ }^{7}$ Cfr. l'intervista dell'architetto Stefano Boeri al quotidiano Repubblica il 21 aprile 2020 dal titolo Via dalla città, nei vecchi borghi c'è il nostro futuro e l'intervento all'iniziativa Risorgimento digitale (29 aprile 2020).

\section{Bibliografia}

Bateson G. 1970, Le radici della crisi ecologica, in Id., Verso un'ecologia della mente (1972), trad. it., Adelphi, Milano 1993, pp. 509-515.

Bauman Z. 2009, Intervista sull'identità, a cura di B. Vecchi, Laterza, Roma-Bari.

Cannata M. (a cura di) 2020, La città per l'uomo ai tempi del Covid-19, La nave di Teseo, Milano.

Castiglioni B. 2010, Educare al paesaggio, trad. it. del report Education on Landscape for Children presentato nel 2009 al Consiglio d'Europa, Museo di Storia Naturale e Archeologia di Montebelluna, Treviso.

Castiglioni B., Cisani M. 2020, The complexity of landscape ideas and the issue of landscape democracy in school and non-formal education: exploring pedagogical practices in Italy, «Landscape Research», 7 aprile 2020, published online. Cepollaro G., Mori L. 2018, Mettersi al mondo. Educazione al paesaggio per le nuove generazioni, ETS, Pisa.

Gattullo M. 2016, Il paesaggio: un bene di tutti, un bene di nessuno o un bene comune?, «Annali del dipartimento di metodi e modelli per l'economia il territorio e la finanza», n. 2, Pàtron, Bologna.

Georgescu-Roegen N. 1982, Energia e miti economici, trad. it., Boringhieri, Torino.

Hargreaves A. 2003, Teaching in the Knowledge Society: Education in the Age of Insecurity, Open University Press, Buckingham.

Iacono A. M. 2010, L'illusione e il sostituto. Riprodurre, imitare, rappresentare, Bruno Mondadori, Milano.

Jaspers K. 1950, Psicologia delle visioni del mondo, trad. it., Astrolabio, Roma.

Lingiardi V. 2017, Mindscapes. Psiche nel paesaggio, Raffaello Cortina, Milano.

Meadows D. H., Meadows D. L., Randers J., Behrens III W. 
W. 1972, The Limits to Growth; trad. it., I limiti dello sviluppo. Rapporto del System Dynamics Group (MIT), per il progetto del Club di Roma sui dilemmi dell'umanità, Mondadori, Milano 1972.

Meadows D. H., Meadows D. L., Randers J. 2004, Limits to Growth: The 30-Year Update, trad. it., I nuovi limiti dello sviluppo, Mondadori, Milano 2006.

Morelli U. 2011, Mente e paesaggio. Una teoria della vivibilità, Bollati Boringhieri, Torino.

Mori L. 2020, Paesaggi utopici. Un manifesto intergenerazionale sulla vivibilità, ETS, Pisa.

Parascandolo F., Tanca M. 2015, Is landscape a commons? Path towards a metabolic approach, in Castiglioni B., Parascandolo F., Tanca M. (a cura di), Landscape as mediator, landscape as commons: international perspectives on landscape research, CLEUP, Padova, pp. 29-45.

Pedroli B., Van Mansvelt J.D. 2006, Awareness-raising, training and education, in Council of Europe, Landscape and sustainable development: challenges of the European landscape Convention, Council of Europe Publishing, Strasbourg.

Sciascia G. (a cura di) 2020, Fabbricare fiducia al tempo del Covid19 e oltre, Rubbettino, Soveria Mannelli.

Settis S. 2010, Paesaggio Costituzione Cemento. La battaglia per l'ambiente contro il degrado civile, Einaudi, Torino.

Tagliagambe S. 2018, II paesaggio che siamo e che viviamo, Castelvecchi, Roma.

Testa A. 2020, L'emergenza climatica è una pandemia al rallentatore, 11 maggio 2020, https://www.internazionale.it/opinione/annamaria-testa/2020/05/11/emergenza-climatica-altra-pandemia

Vitale S. 2015, Il paesaggio e il suo rovescio. Distanza e proiezione nel luogo dell'Altro, Clinamen, Firenze. 